\title{
Phase-Locking of Vortex Lattices Interacting with Periodic Pinning
}

\author{
Charles Reichhardt, Richard T. Scalettar, and Gergely T. Zimányi \\ Department of Physics, University of California, Davis, California 95616. \\ Niels Grønbech-Jensen \\ Department of Applied Science, University of California, Davis, California 95616. \\ NERSC, Lawrence Berkeley National Laboratory, Berkeley, California 94720.
}

(April 6, 2018)

\begin{abstract}
We examine Shapiro steps for vortex lattices interacting with periodic pinning arrays driven by $\mathrm{AC}$ and DC currents. The vortex flow occurs by the motion of the interstitial vortices through the periodic potential generated by the vortices that remain pinned at the pinning sites. Shapiro steps are observed for fields $B_{\phi}<B<2.25 B_{\phi}$ with the most pronounced steps occurring for fields where the interstitial vortex lattice has a high degree of symmetry. The widths of the phase-locked current steps as a function of the magnitude of the AC driving are found to follow a Bessel function in agreement with theory.

PACS numbers: 74.60.Ge, 74.60.Jg
\end{abstract}

Vortex lattices interacting with periodic pinning arrays show a wide range of interesting commensurability or matching effects when the number of vortices is a multiple or rational-multiple of the number of pinning sites. These pinning arrays can be created with lithographic techniques in which arrays of microholes or "antidots" [1] 3] and magnetic dot arrays [4] can act as pinning sites. For small pinning sites only one vortex is trapped on a site as observed in transport measurements [2], Lorentzmicroscopy experiments [3] and simulations [5]. Additional vortices sit in the areas between the pins and under the influence of an applied driving force they can flow between the vortices that have remained trapped at the pinning sites [2, 3.,6] The flowing interstitial vortices experience a periodic potential caused by the repulsive interactions from the vortices at the pinning sites. The motion of the driven interstitial vortices is then analogous to an over-damped particle moving down a tilted washboard. With the addition of an AC driving current, interference effects in the form of Shapiro steps can be expected to occur when the frequency of the particles moving over the washboard matches with one of the harmonics of the driving frequency [7]. Recently Shapiro steps have been observed for driven vortices moving in samples with a periodic array of pinning sites at twice the matching field $B=2 B_{\phi}$ [10] where $B_{\phi}$ is the field for which there is one vortex per pinning site. The height of these current steps (range of phase-locking) strongly suggests that the vortex motion consists of the interstitial vortices moving in the periodic potential from the pinned vortices. Shapiro steps have also been observed by Martinoli et al. [9] for vortices moving over a one dimensional periodic potential created from a periodic thickness modulation. It has further been proposed that Shapiro steps can be seen for vortices in driven flux-transformers.

In this work we investigate numerically and analyti- cally the Shapiro steps for driven vortices in thin film superconductors with periodic pinning arrays. The vortex lattice consists of the pinned vortices at the pinning sites and the sublattice of vortices that sit in the interstitial region. As a function of increasing drive we observe the interstitial vortices moving in one dimensional channels between the pinning sites. With a superimposed AC drive we observe Shapiro steps. We find that for certain commensurate fields, such as $B=2 B_{\phi}$, the system can be modeled as an overdamped driven pendulum with the associated phase locking. We find numerically that the widths of the steps depend on the magnitude of the $\mathrm{AC}$ driving as a Bessel function in agreement with theory. The Shapiro steps are most pronounced for highly symmetric interstitial vortex lattice arrangements. For $B>2 B_{\phi}$ the steps vanish out due to complicated vortex configurations leading to nontrivial flow patterns.

We numerically integrate the overdamped equation of motion for a vortex $i$

$$
\mathbf{f}_{i}=\mathbf{f}_{i}^{v v}+\mathbf{f}_{i}^{v p}+\mathbf{f}_{d}+\mathbf{f}_{a c}=\mathbf{v}_{i} .
$$

Here the total force acting on vortex $i$ is $\mathbf{f}_{i}$. The vortexvortex interaction potential is logarithmic, $U_{v}=-\ln (r)$, and the force on vortex $i$ from all the other vortices is $\mathbf{f}_{i}^{v v}=\sum_{j \neq i}^{N_{v}} \nabla_{i} U_{v}\left(r_{i j}\right)$ [1]. We impose periodic boundary conditions and evaluate the periodic long-range logarithmic interaction with an exact and fast converging sum 112]. The pinning is modeled as attractive parabolic wells with $f_{i}^{v p}=\left(f_{p} / r_{p}\right) \Theta\left(\left|\mathbf{r}_{i}-\mathbf{r}_{k}^{(p)}\right| / \lambda\right) \hat{\mathbf{r}}_{i k}^{(p)}$. Here, $\lambda=1, \Theta$ is the step function, $\mathbf{r}_{k}^{(p)}$ is the location of pinning site $k, f_{p}$ is the maximum pinning force and $\hat{\mathbf{r}}_{i k}^{(p)}=\left(\mathbf{r}_{i}-\mathbf{r}_{k}^{(p)}\right) /\left|\mathbf{r}_{i}-\mathbf{r}_{k}^{(p)}\right|$. The pinning is placed in a rectangular array $\left(L_{x}, L_{y}\right)$ with the ratio of the pinning radius $r_{p}$ to pinning lattice constant $L_{y}$ being $r_{p} / L_{y}=0.164$, close to the ratio 0.2 used in the exper- 
iments [10]. The pinning is placed in a $4 \times 4$ array and the initial vortex configurations are obtained by annealing from a high temperature state where the vortices are liquid and cooling to $T=0$. For certain parameters we have also considered simulations for pinning arrays up to $10 \times 10$ and found only minor differences. We only consider the case for $B>B_{\phi}$ so that the vortex motion will be strictly from the flow of interstitial vortices. The driving force $\mathbf{f}_{d}$ represents the Lorentz force from an applied current. We gradually increment $\mathbf{f}_{d}$ from zero simulating each DC current value for 17500 time steps (the normalized time step is $d t=0.003072$ ) to obtain the average of the vortex velocities. The resulting DC force-velocity curve is proportional to the DC current-voltage curve. The $\mathrm{AC}$ offset is added as $f_{a} \cos (\omega t)$. We conduct a series of simulations where the amplitude $f_{a}$ is varied. In this work both the DC and AC driving forces will be in the $x$-direction.

We first consider the $B=2 B_{\phi}$ case where the interstitial vortices form a perfectly ordered square sub-lattice. The vortex trajectories above depinning are shown in the upper inset of Fig. 1 for this case. Here the interstitial vortices travel in one dimensional paths between the pinned vortex sub-lattice. Further, the moving interstitial vortex lattice retains the same square symmetry as the pinned interstitial vortex lattice. Fig. 1 shows typical simulation results of the voltage response $V_{x}=$ $\left(1 / N_{v}\right) \sum_{i=1}^{N_{v}} \hat{\mathbf{v}}_{i} \cdot \hat{\mathbf{x}}$ versus an applied DC driving force at several different $\mathrm{AC}$ amplitudes for $B=2 B_{\phi}$. The simulation parameters are $\omega=1.6276$ and $L_{x}=L_{y}=1.83$. For zero AC driving the vortex velocities increase linearly with the DC driving force. With applied AC driving there are clear steps where the vortex velocities remain constant for a finite range of DC driving, indicative of phase-locking of the vortex motion. The widths of the steps depend on the magnitude of the $\mathrm{AC}$ drive.

In order to demonstrate that the phase-locking of the interstitial vortex motion is indeed closely related to the well-known Shapiro steps in the AC driven pendulum equation [8] we first make the observation from the inset in Fig. 1 that the interstitial vortices are moving onedimensionally along the $\mathrm{x}$-direction at the symmetry line between the pinned vortices $y=\frac{L_{y}}{2}$, where $L_{y}$ is the distance between two pinning centers along the y-direction. This allows us to write the equation of motion for the unpinned vortices as,

$$
\frac{d}{d t} x_{i}-f_{i}^{v v}\left(x, y=\frac{L_{y}}{2}\right)=f_{d}+f_{a c},
$$

where we have neglected the pinning interaction and motion in the transverse direction. We will make the additional assumptions that unpinned vortices form a perfect rectangular lattice, meaning that they effectively do not interact due to symmetry, and that the pinned vortices are effectively pinned exactly to their pinning site; i.e., that the pinned vortices have no dynamics and form a perfect rectangular lattice with dimensions $L_{x}$ and $L_{y}$.

Under these assumptions each moving vortex obeys the following equation of motion [12]:

$$
\frac{d}{d t} x_{i}-\frac{\pi}{L_{x}} \sum_{k} \frac{\sin \left(2 \pi \frac{x}{L_{x}}\right)}{\cosh \left(2 \pi \frac{L_{y}}{L_{x}}\left(k+\frac{1}{2}\right)\right)-\cos \left(2 \pi \frac{x}{L_{x}}\right)}=f_{d}+f_{a c} .
$$

Considering only the leading term in the above sum, we can simplify the interaction between pinned and unpinned vortices to yield the equation,

$$
\frac{d}{d t} x-\frac{2 \pi}{L_{x}} \operatorname{sech}\left(\pi \frac{L_{y}}{L_{x}}\right) \sin \left(2 \pi \frac{x}{L_{x}}\right)=f_{d}+f_{a} \cos (\omega t),
$$

where we have considered only the contributions, $k=$ $-1,0$, and allowed for a relative error in the force of $\sim \operatorname{sech}\left(\pi \frac{L_{y}}{L_{x}}\right)$, which is obviously small as long as $L_{y} / L_{x}$ is not small. This equation describes the driven overdamped pendulum, and we can therefore apply the procedure for evaluating phase-locking ranges between a pendulum and an AC drive.

Assuming phase-locking where the pendulum (vortex) moves with a frequency $n \omega$, we insert the following ansatz (valid for large AC amplitudes) into the above equation, $x(t)=x_{0}+n \omega \frac{L_{x}}{2 \pi} t+\frac{f_{a}}{\omega} \sin \omega t$, and equate the DC components of the resulting expression, yielding the relationship between the applied AC force and the phase, $2 \pi x_{0} / L_{x}$, for a given integer $n$ :

$$
n L_{x} \frac{\omega}{2 \pi}-\frac{2 \pi}{L_{x}} \operatorname{sech}\left(\pi \frac{L_{y}}{L_{x}}\right) J_{n}\left(\frac{2 \pi f_{a}}{\omega L_{x}}\right) \sin \left(2 \pi \frac{x_{0}}{L_{x}}\right)=f_{d},
$$

where $J_{n}$ is the $n$th order Bessel function of the first kind. The size of the range, $\Delta f_{d}$, in $f_{d}$ for which the vortex motion may stay locked to the AC drive's $n$th harmonic is then given by the extreme values of $\sin \left(2 \pi \frac{x_{0}}{L_{x}}\right)$ :

$$
\Delta f_{d}=\frac{4 \pi}{L_{x}} \operatorname{sech}\left(\pi \frac{L_{y}}{L_{x}}\right)\left|J_{n}\left(\frac{2 \pi f_{a}}{\omega L_{x}}\right)\right| .
$$

By conducting a series of simulations with different AC driving amplitudes we can compare our simulation results for the dependence of the step widths with those predicted from equation (6). In Fig. 2a we plot the widths of the locking ranges for the harmonics, $n=0,1,2$, predicted for our parameters from equation (6) (solid lines) and the widths of the simulated locking ranges, $n=0(\bullet)$, $n=1(\circ)$, and $n=2(\square)$. There is very good agreement between the simulation data and the predicted curves. We note that although equation (6) is for a single interstitial moving vortex at $B=2 B_{\phi}$ the interstitial vortex lattice is symmetric (see Fig. 1a) so the interstitialinterstitial vortex interactions cancel. We also obtain good agreement for the predicted widths from equation (6) and the simulations for the higher harmonics $n>2$ which are not shown here. We note that the agreement 
between the simulation data and the predicted behavior is not expected to be exact since the force that the interstitial vortices experience from the pinned vortex lattice is not strictly sinusoidal. We have also tested equation (6) for different ratios of $L_{x} / L_{y}$ by considering a rectangular pinning array with $L_{x} / L_{y}=2$. The ratio of the step widths for the different directions is $\approx 52$ in good agreement with the theoretical prediction of $\approx 57$. The agreement is still good when we compare the simulated ranges of phase-locking with those predicted by equation (6) for the same parameters as above, but with $L_{x}=2 L_{y}=3.66$. Since the vortices are forced in the $\mathrm{x}$-direction, this is a case where the harmonic potential approximation made in equation (4) is not expected to be as good as for the square lattice case, $L_{x}=L_{y}$. Figure $2 \mathrm{~b}$ shows that simulations at the second matching field, $B=2 B_{\phi},(\square$ and $\circ)$ show less than predicted ranges of locking suggesting that assumptions in the analysis are not well within validity. However, performing the same simulations, but at the matching field with one additional interstitial vortex (filled markers), reveals locking-ranges very close to what is predicted. This underlines that the harmonic potential assumption made in equation (4) is reasonable even for $L_{x}=2 L_{y}$. Closer examination of the dynamics at $B=2 B_{\phi}$ (open markers) shows that internal modes in the moving vortex lattice are being excited and the assumption of cancelation of interstitial vortex interactions become invalid, which is responsible for the deviation between simulations and our prediction in figure $2 \mathrm{~b}$ for $B=2 B_{\phi}$. It is, of course, important to emphasize that the overall features of the locking range is still predicted well by equation (6).

The above analysis suggests that whenever the interstitial vortex lattice is rectangular and the interstitial vortex interactions therefore cancel, Shapiro steps should be observed and be well approximated by equation (6) when $\operatorname{sech}\left(\pi L_{y} / L_{x}\right)$ is small. Square interstitial vortex arrangements are found at $B / B_{\phi}=2,1.5,1.25,1.0625$. For other filling fractions the interstitial vortex lattice is not symmetrical and the interstitial-interstitial interactions do not cancel, leading to some deviations from the predicted phase-locking (the locking range is usually smaller than predicted). This is illustrated in Fig. 3 where we show the widths of the Shapiro steps for different filling fractions for a fixed $\mathrm{AC}$ amplitude and frequency. The Shapiro step widths for the different symmetrical vortex configurations at $B / B_{\phi}=2,1.5$ and 1.0625 are essentially identical. For $B / B_{\phi}=1.375$, and 1.68 the steps are considerably reduced and some fractional Shapiro steps also appear. We find in general that for $B_{\phi}<B<2 B_{\phi}$, the filling fractions that produce square interstitial vortex lattices have the same Shapiro step widths as at $B=2 B_{\phi}$.

Interestingly for $B>2 B_{\phi}$ we find that the step widths remain the same as at $B=2 B_{\phi}$; however, there is a component in $V_{x}$ of the steps that linearly increases with increasing $f_{d}$. For increasing magnetic fields this linear increase in $V_{x}$ of the steps increases until $B \geq 2.25 B_{\phi}$, when the steps can no longer be discerned. This linear increase suggests that only a portion of the vortices are phase locked. The images (not shown) from the simulations suggest that the extra vortices which have been added to the $B=2 B_{\phi}$ sub-lattice cause an additional soliton-like motion which moves at a different speed than the interstitial vortices. To examine this we plot in figure 4 the time dependent vortex velocities for two separate interstitial vortices along the same row at the $n=1$ step $\left(f_{d}=0.39\right)$. In Figs. $4 \mathrm{a}$ and $4 \mathrm{~b}$ for $B=2 B_{\phi}$ the signals for the two particles are identical indicating that the vortices are moving in phase. In Figs. 4c and 4d we plot the signal from a row containing an extra vortex for $B=2.0625 B_{\phi}$. Here the same oscillation as in Figs. $4 \mathrm{a}$ and $4 \mathrm{~b}$ is seen, indicating that phase-locking is occurring; however, there is an additional lower frequency oscillation superimposed. The soliton like nature of this disturbance can be seen by noting this extra oscillation out of phase between the two vortices; similar to a kink soliton on a Frenkel-Kontorova chain [14].

In conclusion, we have observed Shapiro steps in the current-voltage characteristics of driven vortex lattices interacting with periodic pinning. At $B=2 B_{\phi}$ where the vortex motion consists of the one dimensional flow of interstitial vortices between the pinned vortices, Shapiro steps are observed in agreement with recent experiments [10]. We show that for certain filling fractions the equation of motion for a driven interstitial vortex with a drive can be mapped to a driven overdamped pendulum. We derive the widths of the Shapiro steps as a function of relevant experimental parameters, and find excellent agreement between theory and simulations. For filling fractions where interstitial-interstitial vortex interactions become relevant the step widths are reduced. For $B>2 B_{\phi}$ the steps begin to vanish due to an additional soliton like flow and other dynamical complexity.

Acknowledgments: We thank C.J. Olson for critical reading of this manuscript. This work was supported by the Director, Office of Advanced Scientific Computing Research, Division of Mathematical, Information, and Computational Sciences of the U.S. Department of Energy under contract number DE-AC03-76SF00098 as well as CLC and CULAR (Los Alamos National Laboratory).

[1] M. Baert et al., Phys. Rev. Lett. 74, 3269 (1995); J.Y. Lin et al., Phys. Rev. B 54, R12 714 (1996); A. Bezryadin, Yu. B. Ovchinnikov, and B. Pannetier, Phys. Rev. B 53, 8553 (1996); A. Castellanos et al., Appl. Phys. Lett. 71, 962 (1997); V.V. Metlushko et al., Europhys. Lett. 41, 333 (1998); V.V. Moshchalkov et al., Phys. Rev. B 57, 3615 (1998); V.V Metlushko et al., Phys. Rev. B 59, 603 (1999).

[2] E. Rosseel et al., Phys. Rev. B 53, R2983 (1996). 
[3] K. Harada et al., Science 271, 1393 (1996).

[4] J.I. Martín et al., Phys. Rev. Lett. 79, 1929 (1997); D.J. Morgan and J.B. Ketterson, Phys. Rev. Lett. 80, 3614 (1998); Y. Jaccard et al., Phys. Rev. B, 58, 8232 (1998). M.J. Van Bael et al., Phys. Rev. B 59, 14674 (1999); J.I. Martín, et al., Phys. Rev. Lett. 83, 1022 (1999).

[5] C. Reichhardt, C.J. Olson and F. Nori, Phys. Rev. B 57, 7937 (1998).

[6] C. Reichhardt, C.J. Olson, and F. Nori, Phys. Rev. Lett. 78, 2648 (1997).

[7] S. Shapiro, Phys. Rev. Lett. 11, 80 (1963).

[8] A. Barone and G. Paternó, Physics and Applications of the Josephson Effect (John Wiley, 1982).

[9] P. Martinoli, O. Daldina, C. Leemann, and E. Stocker, Solid State Commun. 17, 205 (1975).

[10] L. Van Look et al., Phys. Rev. B 60, R6998 (1999).

[11] N. Grønbech-Jensen, A.R. Bishop, and D. Domínguez, Phys. Rev. Lett. 76, (1996).

[12] N. Grønbech-Jensen, Int. J. Mod. Phys. C 7, 873 (1996); Comp. Phys. Comm. 119, 115 (1999).

[13] N. Grønbech-Jensen and M. R. Samuelsen, Phys. Lett. A 191, 57 (1994).

[14] P. S. Lomdahl and D. J. Srolovitz, Phys. Rev. Lett. 57, 2702 (1986).

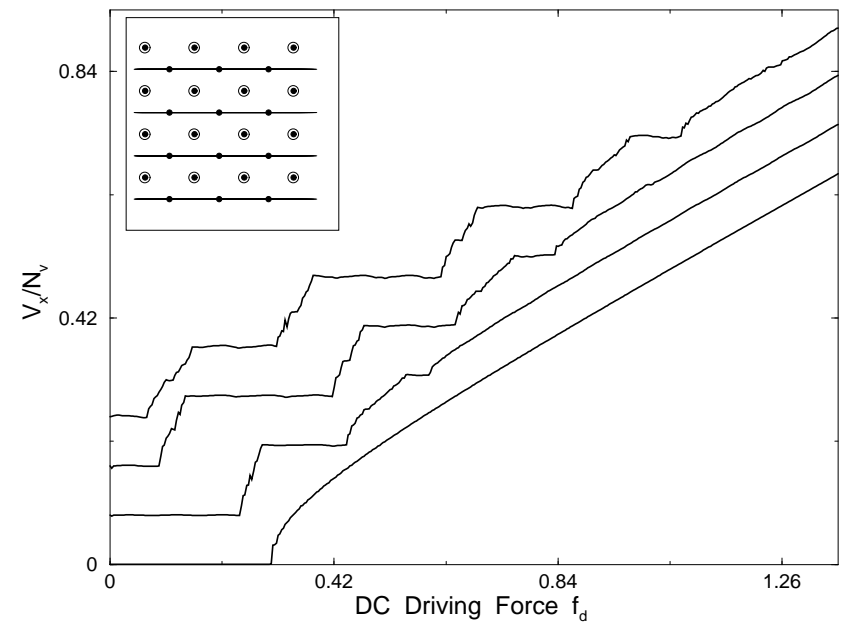

FIG. 1. Average vortex velocity $V_{x}$ versus applied DC driving for varying applied AC driving of (from top to bottom) $f_{a}=0.814,0.5078,0.22135$, and 0 for $B=2 B_{\phi}$. The curves have been shifted up from the 0 curve for presentation. Here clear steps can be seen for finite applied AC drives. Inset: the typical vortex trajectories, positions (black circles) and pinning sites (open circles) from the simulations. Here the motion consists of interstitial vortices moving in one dimensional channels between the vortices pinned at the pinning sites. Parameters are: $\omega=1.6276$ and $L_{x}=L_{y}=1.83$.
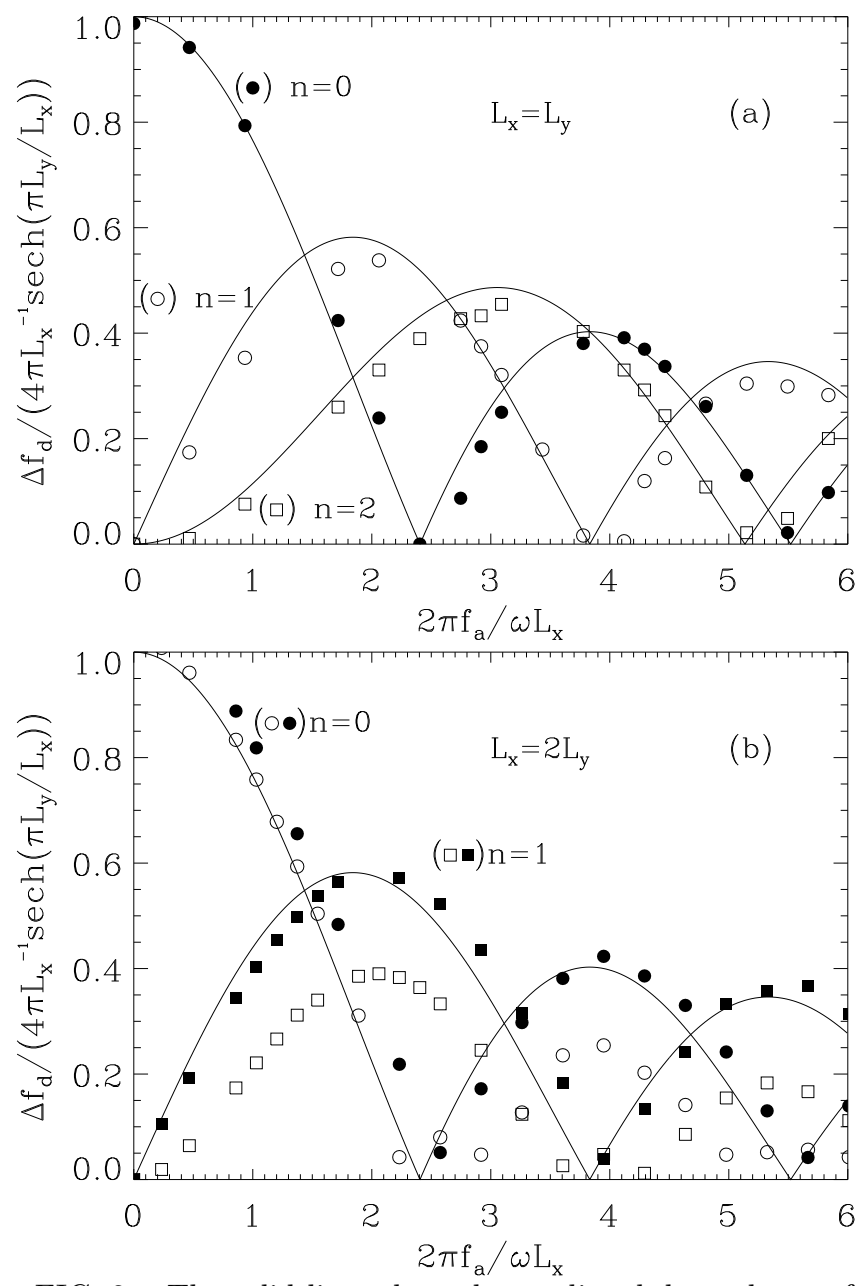

FIG. 2. The solid lines show the predicted dependence of the widths of the steps in the velocity-force curves for varying applied AC drive amplitude from equation (6) for the first three harmonics $n=0, n=1$, and $n=2$. Markers represent the simulation results of the phase-locking range in DC current. $\omega=1.6276$. AC amplitude, $f_{a}$, is varied from $f_{a}=0$ to $f_{a} \approx 2.93 \frac{L_{x}}{L_{y}}$. (a) $L_{x}=L_{y}=1.83$ and $B=2 B_{\phi}$. (b) $L_{x}=2 L_{y}=3.66$; open markers represent simulations at $B=2 B_{\phi}$ and filled makers represent simulations with a single unpinned vortex. 


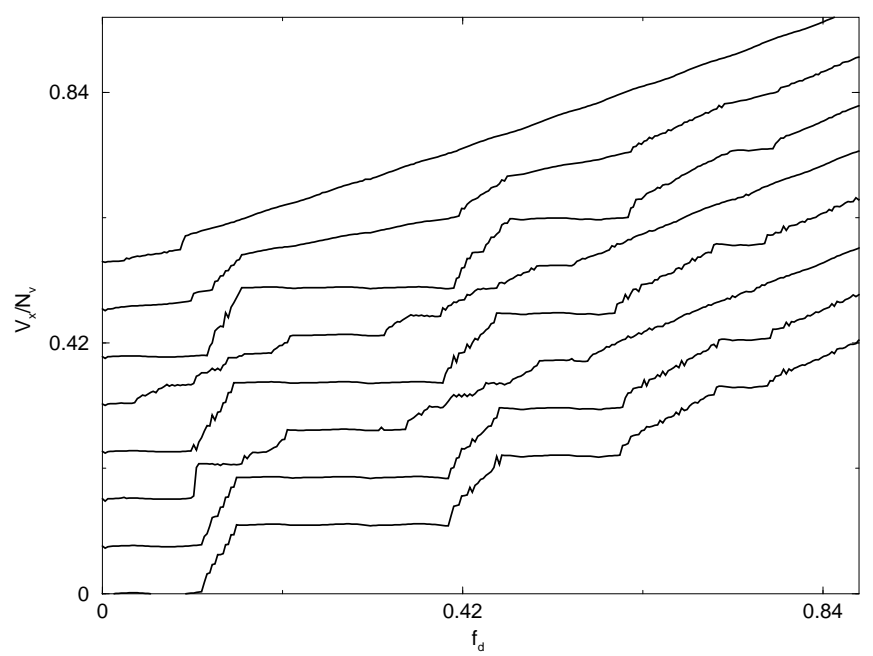

FIG. 3. Average vortex velocity $V_{x}$ versus driving force for constant AC driving $f_{a}=0.5078$ for varying fields (from top to bottom) $B / B_{\phi}=2.25,2.125,2.0168,1.5,1.375,1.25,1.0625$ . Each curve has been shifted for clarity. Parameters are: $\omega=1.6276$ and $L_{x}=L_{y}=1.83$.
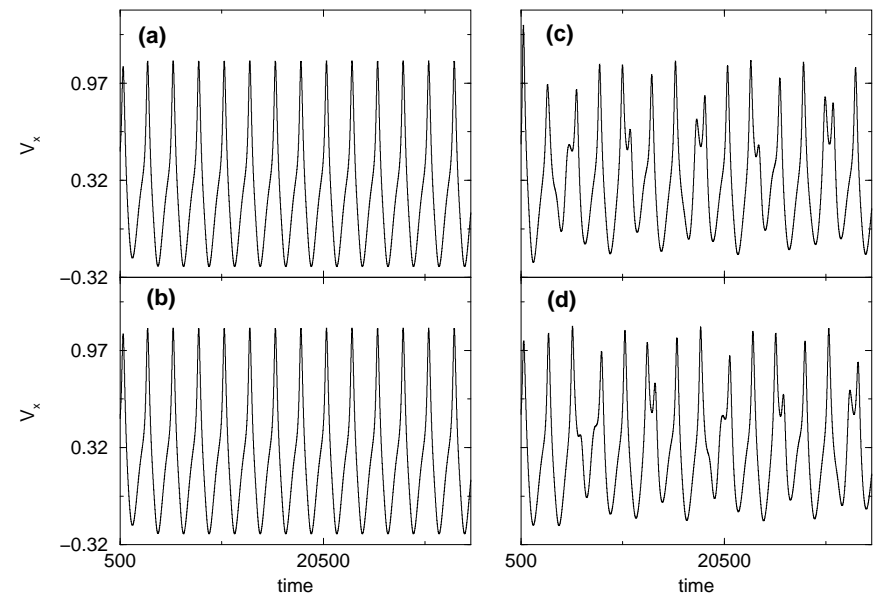

FIG. 4. (a) The time dependent vortex velocity for two different vortices in a single row for $B=2 B_{\phi}$ in the middle of the $n=1$ step for the same system as in Fig. 1. (b) The same for $B=2.0625 B_{\phi}$. Parameters are: $\omega=1.6276$, $L_{x}=L_{y}=1.83, f_{d}=0.38$, and $f_{a}=0.442$. 